\title{
Effect of Chamber Conditions and Substrate Type on PECVD of SiGeSn Films
}

\author{
Venkat Hariharan ${ }^{1}$, Jignesh Vanjaria ${ }^{2}$, Arul Chakkaravarthi Arjunan ${ }^{3 *}$, \\ Gary S. Tompa 3 , Hongbin $\mathbf{Y u}^{4}$ \\ ${ }^{1}$ Logic Technology Development, Intel Corporation, Hillsboro, USA \\ ${ }^{2}$ Transport and Energy, School for Engineering of Matter, Arizona State University, Tempe, USA \\ ${ }^{3}$ Structured Materials Industries Inc., Piscataway, USA \\ ${ }^{4}$ School of Electrical, Computer and Energy Engineering, Arizona State University, Tempe, USA \\ Email: *aarul.smi@gmail.com
}

How to cite this paper: Hariharan, $\mathrm{V}$. Vanjaria, J., Arjunan, A.C., Tompa, G.S. and Yu, H.B. (2021) Effect of Chamber Conditions and Substrate Type on PECVD of SiGeSn Films. Crystal Structure Theory and Applications, 10, 39-56.

https://doi.org/10.4236/csta.2021.103004

Received: July 11, 2021

Accepted: August 27, 2021

Published: August 30, 2021

Copyright (c) 2021 by author(s) and Scientific Research Publishing Inc. This work is licensed under the Creative Commons Attribution International License (CC BY 4.0).

http://creativecommons.org/licenses/by/4.0/

\section{Open Access}

\begin{abstract}
In the past studies have shown that the addition of Ge and Sn into Si lattice to form $\mathrm{SiGeSn}$ enhances its carrier mobility and band-gap properties. Conventionally SiGeSn epitaxial films are grown using Ultra-High Vacuum (UHV) conditions with pressures ranging from $10^{-8}$ torr to $10^{-10}$ torr which makes high volume manufacturing very expensive. On the contrary, the use of lowpressure CVD processes (vacuum levels of $10^{-2}$ torr to $10^{-4}$ torr) is economically more viable and yields faster deposition of SiGeSn films. This study outlines the use of a cost-effective Plasma Enhanced Chemical Vapor Deposition (PECVD) reactor to study the impact of substrate temperature and substrate type on the growth and properties of polycrystalline SiGeSn films. The onset of polycrystallinity in the films is attributed to the oxygen-rich PECVD chamber conditions explained using the Volmer-Weber (3D island) mechanism. The properties of the films were characterized using varied techniques to understand the impact of the substrate on film composition, thickness, crystallinity, and strain.
\end{abstract}

\section{Keywords}

Thin Film Growth, Volmer-Weber Mechanism, Plasma Enhanced Chemical Vapor Deposition, Silicon Photonics, Carrier Mobility, Band-Gap Engineering, Semimetal Alloys

\section{Introduction}

The number of transistors in integrated microelectronic chips has increased exponentially with every tech node thus enabling ultra-high-performance compu- 
ting. But this has also resulted in dramatic increases in the power consumption of these chips due to the drastically higher frequency of on and off operations. Group IV crystals have played a pivotal role in developing the field of integrated silicon photonics. They have also been employed to demonstrate high-performance PMOS devices to enhance carrier mobility of intrinsic silicon for advanced logic transistors [1] [2]. Germanium is an important semiconducting material which has several attractive features like higher carrier mobility than $\mathrm{Si}$, a smaller energy difference between the $\Gamma$ point and the $\mathrm{L} / \mathrm{X}$ point of $136 \mathrm{meV}$, longer cut-off wavelength than $\mathrm{Si}$ (Ge has $1.55 \mu \mathrm{m}$ ) and compatibility with the well-established silicon CMOS process technology. Ge thin films have also been used as passive components, such as waveguides and amplifiers, in integrated photonic circuits [3]. Ge can also act as a buffer for the growth of group IV alloys such as $\mathrm{GeSn}$ and $\mathrm{SiGeSn}$ on $\mathrm{Si}$ substrates to counter the issue of large lattice mismatch between the alloys and the Si substrate.

The alloy of $\mathrm{Sn}$ with SiGe has been a topic of interest as varying the concentration of Sn provides band-gap tunability and variable lattice parameters. Research has shown that these alloys possess a diamond-like crystallinity with equal probability of the constituent elements in each location [4]. Due to this characteristic, the composition of the alloy can be modified without a change in the lattice parameter which results in the tunable band-gaps. The band-gap of the alloy can be varied from $0.6 \mathrm{eV}$ to $1.1 \mathrm{eV}$ by tuning concentrations of $\mathrm{Si}$ and Sn. G. Sun et al. [5] [6] also indicated that the alloy transitions from an indirect to a direct band-gap as the Sn concentration exceeds $13 \%$. Although polycrystalline GeSn and SiGeSn alloys are not employed for photonic applications, their enhanced carrier mobility compared to polycrystalline Si make them attractive candidates in transistors and tandem solar cells [7]. They also find applications in thermoelectric generators due to their low thermal conductivities [8]. Thus, $\mathrm{SiGeSn}$ alloys can play a promising role in a wide variety of applications.

Crystal growth of GeSn and SiGeSn thin films involves several challenges. The first key challenge is the lattice mismatch between elemental $\mathrm{Si}, \mathrm{Ge}$, and $\mathrm{Sn}$. The lattice constant of Sn is $6.493 \AA$, Ge is $5.658 \AA$ and $S i$ is $5.431 \AA$. This mismatch leads to non-homogeneity during film growth that causes severe surface roughening as is the case of Ge heteroepitaxy on $\mathrm{Si}$ (001) substrate with a lattice mismatch of 4.2\% [9] [10]. The second issue is the low solubility of $\alpha$-Sn in Ge and $\mathrm{Si}$. The thermodynamic solubility of $\mathrm{Sn}$ in $\mathrm{Ge}$ is approximately $1.1 \%$ and drops below $1 \%$ closer to the eutectic temperature of $231.1^{\circ} \mathrm{C}$ while it is $0.1 \%$ in $\mathrm{Si}$ at room temperature [11]. Thus, the solubility of $\mathrm{Sn}$ in the alloy significantly decreases with increasing $\mathrm{Si}$ concentration in the alloy. The third major issue is that the semiconducting form of $\mathrm{Sn}(\alpha-\mathrm{Sn})$ is unstable at room temperature and the phase transforms into the metallic form $(\beta$-Sn). Hence it tends to precipitate out of the epilayer passing through a phase transition from its semiconducting phase with a diamond cubic lattice to a metallic phase with a body-centered tetragonal structure [12]. Thus, non-equilibrium growth techniques at low tem- 
peratures process must be employed to deposit GeSn and SiGeSn thin films with Sn concentrations that exceed the solubility in Ge or SiGe.

Several approaches have been used to grow SiGeSn thin films-pulsed laser deposition, sputtering, molecular beam epitaxy and chemical vapor deposition. The highest material quality films thus far have been achieved with Molecular Beam Epitaxy (MBE) and Chemical Vapor Deposition (CVD). Solid and gaseous precursors have been studied for MBE by [13] [14] [15] [16]. Polycrystalline, diamond lattice GeSn with Sn concentrations of up to $30 \%$ were achieved at growth temperatures of $170^{\circ} \mathrm{C}$ using $\mathrm{MBE}$. Ion-assisted $\mathrm{MBE}$ enabled the growth of pseudo-morphic $\mathrm{Ge}_{1-\mathrm{x}} \mathrm{Sn}_{\mathrm{x}}$ epi layers on Ge with $0.035<\mathrm{x}<0.115$ and layer thicknesses between $50 \mathrm{~nm}$ and $200 \mathrm{~nm}$. SiGeSn epilayers with Sn content of $4 \%$ - $15 \%$ and Si content of $20 \%$ - $45 \%$ have also been reported by MBE at growth temperatures of $100^{\circ} \mathrm{C}-250^{\circ} \mathrm{C}$. Although $\mathrm{MBE}$ offers good composition control at low growth temperatures, CVD has been the most used technique to deposit SiGeSn films due to high growth rates, the possibility of selective growth and uniform deposition over large-scale wafers in a cost-effective manner.

At low growth temperatures, the rate of deposition is governed by the surface reactions. The rate is a strong function of precursor flow rates, substrate temperature, and chamber pressure. The kinetics also depends on the design of the CVD tool employed. By choosing and precisely controlling the appropriate growth parameters in different types of CVD reactors, several groups have succeeded in growing metastable, supersaturated, intrinsic and doped GeSn and SiGeSn alloys on Si (001), Ge (001) substrates [17] [18]. The researchers at the Arizona State University developed a hot wall ultra-high vacuum CVD system to deposit films using higher-order silanes and germanes along with stannane $\left(\mathrm{SnD}_{4}\right)$ as the precursors for Si, Ge and Sn [19] [20] [21]. The first growth of SiGeSn ternaries was enabled using GeSn/Si (001) buffers under Ultra-High Vacuum (UHV) conditions and a precursor combination of $\mathrm{SiH}_{3}, \mathrm{GeH}_{3}$ and $\mathrm{SnD}_{4}$. In order to grow on Si (001) directly and on the Ge-buffer layer, a novel precursor combination $\mathrm{SnD}_{4} / \mathrm{Ge}_{2} \mathrm{H}_{6} / \mathrm{Si}_{3} \mathrm{H}_{8}$ had to be introduced and at low growth temperatures, $\mathrm{SiGeH}_{6}$ was added to fine-tune the Si concentration [22]. Following this, efforts were made to use silane, germane and $\mathrm{SnCl}_{4}$ instead of higher-order gases to lower operational expenditure. These efforts have been recently proved successful by the utilization of uniquely designed CVD reactors, such as cold wall ultra-high vacuum CVD, reduced pressure CVD and atmospheric pressure CVD systems [23]-[28]. Si-Ge-Sn CVD advancements in the past two decades have thus enabled superior films for Si photonics and microelectronics.

This work attempts to characterize the less explored plasma-enhanced CVD process that is thermally and economically viable to deposit polycrystalline $\mathrm{Si}$ GeSn films using germane $\left(\mathrm{GeH}_{4}\right)$ for $\mathrm{Ge}$, disilane $\left(\mathrm{Si}_{2} \mathrm{H}_{6}\right)$ for $\mathrm{Si}$ and tin chloride $\left(\mathrm{SnCl}_{4}\right)$ for $\mathrm{Sn}$ as the precursors. PECVD enabled low substrate temperatures and high deposition rates by providing additional energy for precursor bond dissociation through collisions with plasma ions. The effect of temperature and con- 
centration grading were discussed in prior manuscripts [29] [30]. The focal point of this manuscript is the effect of the substrate type on the SiGeSn film properties.

\section{Experimental}

The reactor consisted of a cylindrical quartz chamber with two injection lines at the top for the precursors (one for hydride precursors of silicon and germanium and the other for the Sn precursor), a gas delivery system and a vacuum exhaust system connected through flange at the bottom. The quartz chamber had an inner diameter of 3 inches and a length of $1.5 \mathrm{ft}$. The substrates were placed on a circular molybdenum susceptor within the quartz chamber. The susceptor was 2 inches in diameter and 1/2 inch thick. After the substrate placement on the susceptor, the top flange was attached, and the chamber was evacuated using a rotary vane pump boosted by a root's blower pump. Vacuum levels of up to $10^{-2}$ torr were achievable. Once the vacuum was established, the susceptor was heated using a set of 8 infra-red lamps arranged in a circle around it. The susceptor temperatures were measured using pyrometers. Once the desired temperatures were reached, the precursors were introduced into the reactor through gas inlets. The Si and Ge precursor gases were delivered from the gas cabinet to the reactor chamber via quarter inch pipes. The flow of the precursor gases to the quartz chamber was controlled using mass flow controllers calibrated for each gas. Commercially available $\mathrm{GeH}_{4}\left(5 \%\right.$ in $\left.\mathrm{H}_{2}\right), \mathrm{Si}_{2} \mathrm{H}_{6}\left(10 \%\right.$ in $\mathrm{H}_{2}$ ) and $\mathrm{SnCl}_{4}(99.999 \%)$ were selected as the precursors for $\mathrm{Ge}, \mathrm{Si}$ and $\mathrm{Sn}$ respectively. $\mathrm{As} \mathrm{SnCl}_{4}$ is a liquid at room temperature, a bubbler system was designed for a carrier gas $\left(\mathrm{H}_{2}\right)$ to bubble through the liquid and transport vapors of $\mathrm{SiCl}_{4}$ to the chamber. Plasma was generated in the chamber during film growth using a plasma induction $\mathrm{Cu}$ wire wrapped around the quartz chamber powered by a $250 \mathrm{kHz} 1 \mathrm{~kW}$ RF power source. The schematic and the picture of the reactor are shown in Figure 1.

Buffer-free polycrystalline SiGeSn thin films were deposited on the various substrates using the reactor. Films were grown on c-plane sapphire wafers, 100 $\mathrm{nm} \mathrm{SiO}{ }_{2}$ coated silicon (100) wafers and phosphorus doped $\mathrm{Si}$ (100) wafers (resistivity $10^{-3} \Omega$-cm). The Si substrates were placed in a buffered HF bath for $\sim 5$ min, washed with DI water, blow dried with nitrogen, and immediately transferred to the growth chamber to minimize the growth of native oxide. The insulating substrates viz. sapphire and silicon dioxide on silicon were placed besides the $\mathrm{Si}$ substrate on the susceptor without any pre-deposition cleaning. $\mathrm{GeH}_{4}$ and $\mathrm{Si}_{2} \mathrm{H}_{6}$ flow rates were set at $200 \mathrm{sccm}$ and $50 \mathrm{sccm}$ respectively for runs 1, 2 and 3. For run 4 the $\mathrm{GeH}_{4}$ flow rate was dropped to $50 \mathrm{sccm}$ while $\mathrm{Si}_{2} \mathrm{H}_{6}$ flow rate was still maintained at $50 \mathrm{sccm}$. Films were deposited for 40 minutes at a substrate temperature of $380^{\circ} \mathrm{C}$ and chamber pressure of 1 torr.

Structural and optical properties of the deposited films were studied using Rutherford Backscattering (RBS), X-Ray Diffraction (XRD), Scanning Electron Microscopy (SEM) and Raman spectroscopy. 


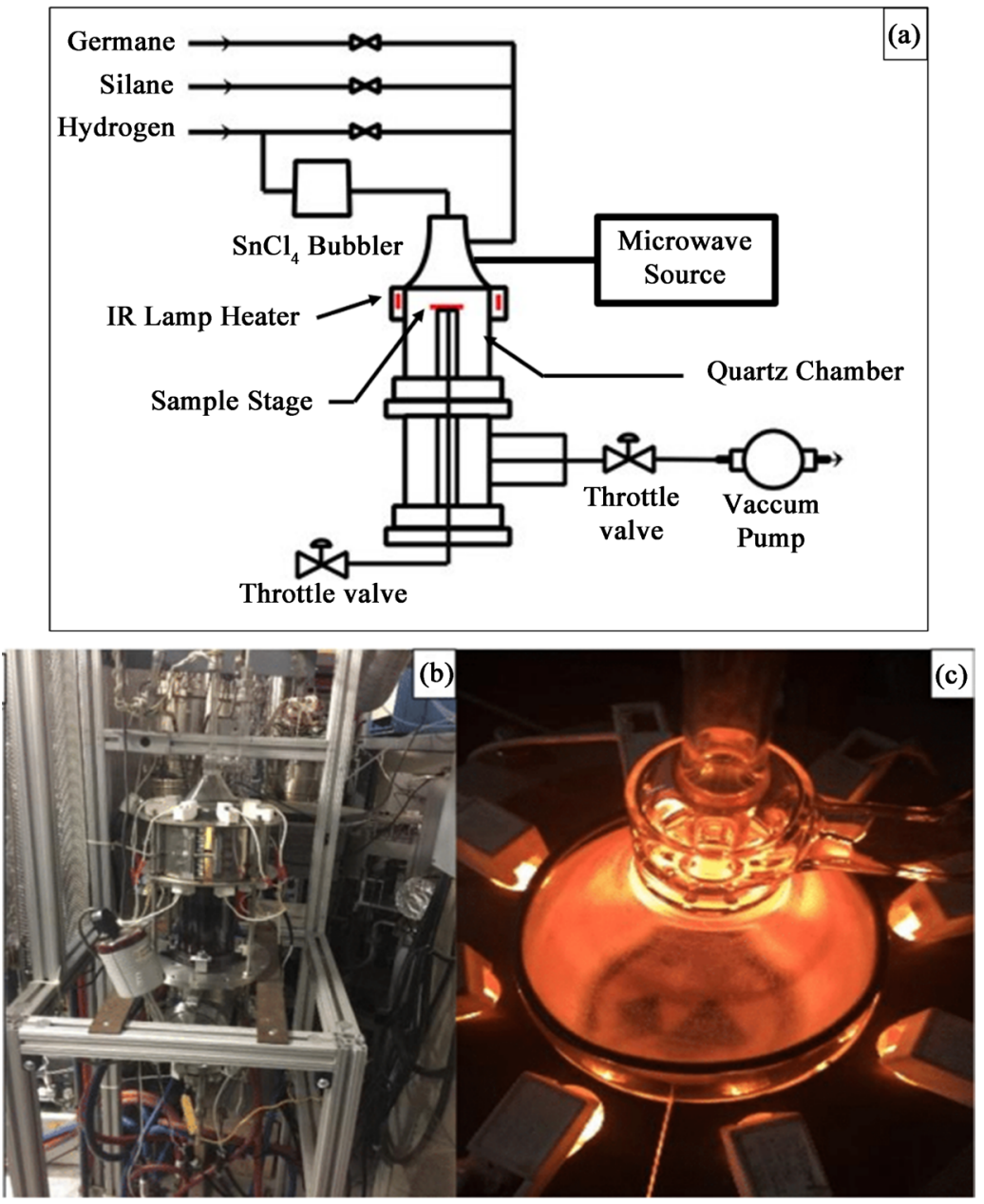

Figure 1. Schematic (a) and pictures ((b), (c)) of the PECVD reactor.

The crystalline quality of the films was analyzed using XRD spectra obtained from PANalytical XPert Pro MRD diffractometer with $\mathrm{Cu} \mathrm{K} \alpha$ radiation. The elemental profile measurements were carried out using a 1.7 MV Cockroft-Walton, gas-insulated high frequency tandem accelerator with a beamline and analysis chamber. $2 \mathrm{MeV} \mathrm{He}^{2+}$ ions were used as the ion source and data collected at $10^{5}$ rotating random counts with $8^{\circ}$ offset between ion beam and sample surface.

Top SEM images were obtained to study the impact of growth temperature on the SiGeSn grain structure using a Hitachi S-4700-II scanning electron microscope.

Raman spectroscopy studies were performed at room temperature using a 532 $\mathrm{nm} 50 \mathrm{~mW}$ green laser coupled to a WiTec Alpha 300R Confocal Raman Imaging system and a Princeton Instruments Acton SP2300 imaging spectrograph to analyze the nature of bonding and strain present in the films.

\section{Results and Discussions}

\subsection{Effect of Temperature on SiGeSn Film}

The effects of temperature on SiGeSn thin film growth were discussed in a prior manuscript to determine the optimal temperatures for film deposition 
[29]. Analysis showed that all the growth temperatures studied $\left(350^{\circ} \mathrm{C}, 380^{\circ} \mathrm{C}\right.$, $400^{\circ} \mathrm{C}$ and $450^{\circ} \mathrm{C}$ ) resulted in polycrystalline films. The results were attributed to the presence of $\mathrm{O}_{2}$ and water vapor inside the chamber during growth due to the non-UHV conditions in the chamber. It was also observed that the temperatures between $350^{\circ} \mathrm{C}$ and $380^{\circ} \mathrm{C}$ resulted in films with structurally uniform lattices with roughnesses seen in the Nanoscale. On the contrary, the higher temperatures of $400^{\circ} \mathrm{C}$ and $450^{\circ} \mathrm{C}$ resulted in the phase separation of $\beta$-Sn. These results were explained by the low thermodynamic solubility of Sn atoms with $\mathrm{Ge}$ and the higher temperatures increasing the mobility of $\mathrm{Sn}$ atoms which makes it thermodynamically more favorable for them to coalesce by the process called Ostwald ripening effect [29]. It was also observed that the lowest temperature of $350^{\circ} \mathrm{C}$ resulted in the highest $\mathrm{Sn}$ concentrations owing to the lowest mobilities of Sn atoms at these temperatures. However, a higher thickness was observed at $380^{\circ} \mathrm{C}$ compared to $350^{\circ} \mathrm{C}$. It was concluded that the higher temperature favored higher precursor cracking rates thus leading to a faster rate of thin film deposition. Thus, $380^{\circ} \mathrm{C}$ was selected as the substrate temperature to study the impact of substrate type in the next section. The surface morphology of the films obtained at the various temperatures is provided in Figure 2.

\subsection{Effect of Substrate on SiGeSn Film}

Figure 3(a) shows the RBS spectrum of a film deposited on sapphire substrate.

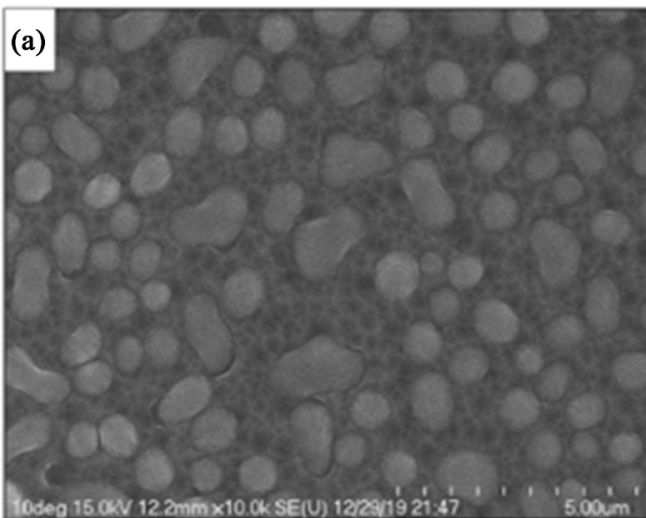

(b)

(c)

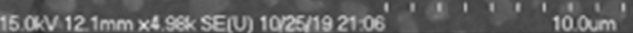

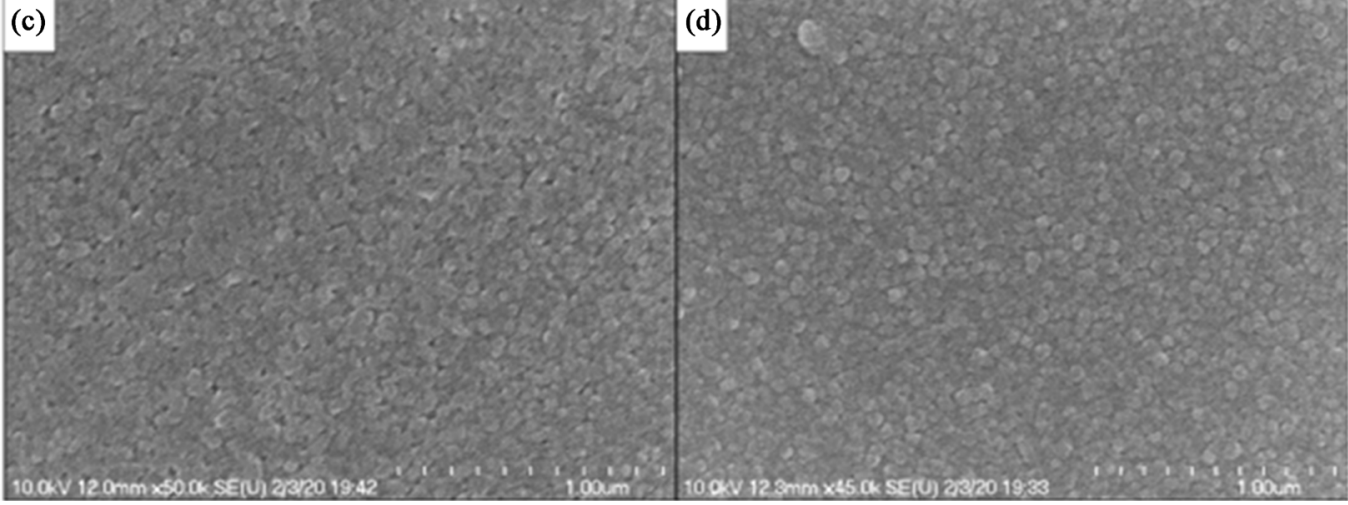

Figure 2. Top down SEM images of SiGeSn films grown at (a) $450^{\circ} \mathrm{C}$, (b) $400^{\circ} \mathrm{C}$, (c) $380^{\circ} \mathrm{C}$, (d) $350^{\circ} \mathrm{C}-$ Ostwald ripening seen for runs (a) and (b). 
The black line indicates the data obtained from RBS while the red line indicates the simulation fitted for the data using RUMP software. The position of the edges in the spectrum corresponds to the different elements and their height is related to their atomic concentrations. The signal from the different elements present in the film i.e., $\mathrm{Si}, \mathrm{Ge}$, and $\mathrm{Sn}$, take the form of plateaus which indicate the elements are uniformly distributed throughout the films. The thicknesses of the films were estimated by dividing the observed surface atomic density with the weighted volumetric atomic density as derived from the extracted compositions. The composition and the thickness obtained from RBS studies for grown films at different growth conditions are given in Table 1. Figure 3(b) is an overlay of RBS spectra with film grown on two different substrates, sapphire, and silicon dioxide.

The analysis of the RBS spectra of the grown films indicated that $\mathrm{Sn}$ and $\mathrm{Si}$ concentrations up to $7 \%$ and $50 \%$ respectively were realized. Also, the use of

Table 1. Growth conditions and film compositions and thicknesses calculated using RBS.

\begin{tabular}{|c|c|c|c|c|c|c|c|c|c|c|}
\hline $\begin{array}{l}\text { Run } \\
\text { No. }\end{array}$ & $\begin{array}{c}\mathrm{Si}_{2} \mathrm{H}_{6} \\
\text { flow } \\
\text { rate } \\
(\mathrm{sccm})\end{array}$ & $\begin{array}{c}\mathrm{GeH}_{4} \\
\text { flow } \\
\text { rate } \\
(\mathrm{sccm})\end{array}$ & $\begin{array}{c}\mathrm{SnCl}_{4} \\
\text { bubbler } \\
\text { flow rate } \\
(\mathrm{sccm})\end{array}$ & $\begin{array}{c}\mathrm{SnCl}_{4} \\
\text { bubbler } \mathrm{t} \\
\text { emperature } \\
\left({ }^{\circ} \mathrm{C}\right)\end{array}$ & $\begin{array}{c}\mathrm{H}_{2} \\
\text { flow } \\
\text { rate } \\
(\mathrm{sccm})\end{array}$ & Substrate & $\mathrm{Si} \%$ & $\mathrm{Ge} \%$ & Sn $\%$ & $\begin{array}{c}\text { Film } \\
\text { thickness } \\
(\mathrm{nm})\end{array}$ \\
\hline \multirow{2}{*}{1} & \multirow{2}{*}{50} & \multirow{2}{*}{200} & \multirow{2}{*}{25} & \multirow{2}{*}{20} & \multirow{2}{*}{150} & Sapphire & 8.5 & 85.0 & 6.5 & 607 \\
\hline & & & & & & $\mathrm{SiO}_{2}$ & 8.4 & 84.9 & 6.7 & 583 \\
\hline \multirow{2}{*}{2} & \multirow{2}{*}{50} & \multirow{2}{*}{200} & \multirow{2}{*}{15} & \multirow{2}{*}{20} & \multirow{2}{*}{150} & Sapphire & 25.6 & 71.9 & 2.5 & 234 \\
\hline & & & & & & $\mathrm{SiO}_{2}$ & 25.9 & 71.3 & 2.8 & 229 \\
\hline \multirow{2}{*}{3} & \multirow{2}{*}{50} & \multirow{2}{*}{200} & \multirow{2}{*}{25} & \multirow{2}{*}{10} & \multirow{2}{*}{150} & Sapphire & 11.6 & 84.9 & 3.5 & 626 \\
\hline & & & & & & $\mathrm{Si}$ & 12.0 & 84.0 & 4.0 & 616 \\
\hline \multirow{2}{*}{4} & \multirow{2}{*}{50} & \multirow{2}{*}{50} & \multirow{2}{*}{20} & \multirow{2}{*}{15} & \multirow{2}{*}{150} & Sapphire & 53.0 & 45.0 & 2.0 & 284 \\
\hline & & & & & & $\mathrm{Si}$ & 53.9 & 44.3 & 1.8 & 279 \\
\hline
\end{tabular}

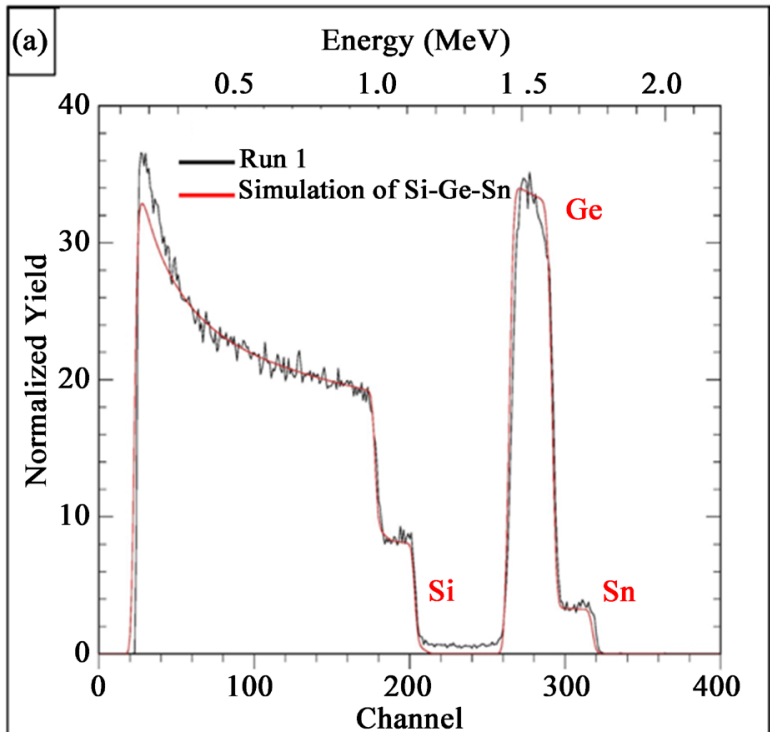

(b)
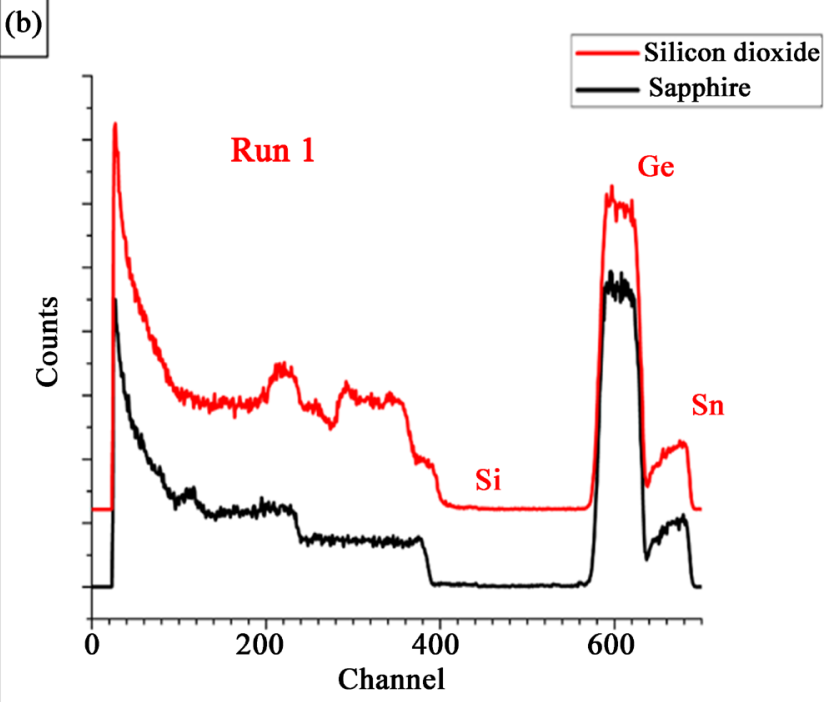

Figure 3. (a) RBS raw spectrum and simulation of SiGeSn film grown on sapphire substrate; (b) RBS spectra overlay of SiGeSn films with similar growth conditions grown on silicon dioxide and sapphire. 
different substrates was seen to not affect the composition or thickness of the deposited films. It is theoretically proven that equilibrium is reached after the first few atomic layers of SiGeSn are grown and hence the type of substrate has no influence on the bulk composition and thicknesses [31]. The reactions and the atomic transport theories at the interface are discussed later in this paper. Further, increasing the $\mathrm{SnCl}_{4}$ bubbler flow rate (Runs 1 and 3) or bubbler temperature (Runs 1 and 2) increases the Sn concentration due to the higher flux of Sn precursor reaching the substrate. Also, as the Sn content in the film increases, the Si content decreases correspondingly. This is attributed to Si and Sn atoms competing for positions in the Ge lattice matrix and higher substitutional $\mathrm{Sn}$ leading to limited lattice sites for Si atoms [25]. Thus, the compositions of the films can be finely tuned by the relative flows of the precursors in the gas.

The Raman spectra of the films with varying composition deposited on the different substrates (Runs 2 and 4 from Table 1) are shown in Figure 4. Peaks correlating to the Ge-Ge bond and Si-Ge bond are seen in all spectra while the peak correlating to the Ge-Sn bond are only seen as minor shoulders to the Ge-Ge peaks. This is due to the low concentration of Sn in these films. Additionally, the peaks in all the spectra shift towards lower wavenumbers than their expected positions implying the incorporation of $\mathrm{Sn}$ in the films. Thus, the presence of the bonds and their red shift confirms the formation of the ternary component in the deposited films. The magnitude of red shift of the Raman peaks of different films is determined by the strain arising from the thermal stress due to differences in the coefficients of thermal expansion between the

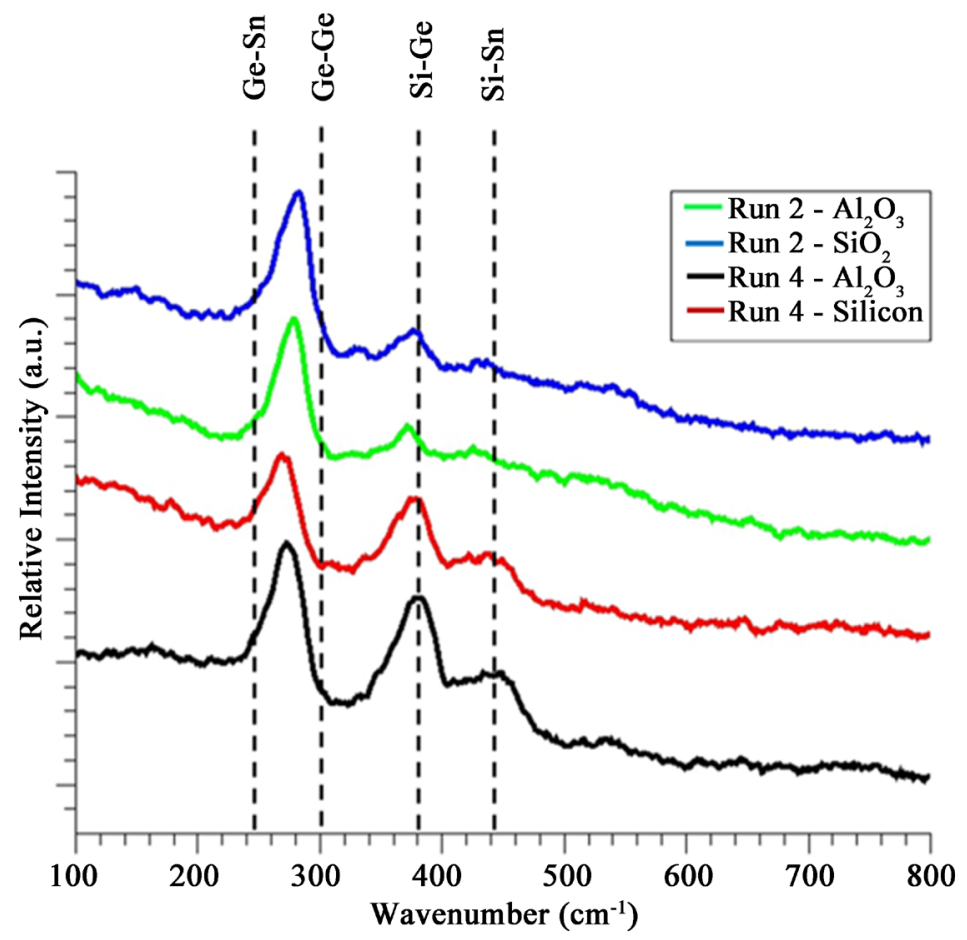

Figure 4. Raman spectra of SiGeSn films with varying compositions on different substrates. 
film and substrate as well as the differences in the lattice constants between $\mathrm{Si}$, $\mathrm{Ge}$ and $\mathrm{Sn}$ while the disorder is due to the various lattice imperfections present in the polycrystalline film such as grain boundaries and misfit dislocations. It is seen that, as Si:Ge precursor ratio increases, the concentration of $\mathrm{Si}-\mathrm{Sn}$ bonds go up as seen for runs 4 . It is also seen that; the substrate type does not play a significant role in the intensity of each type of bond. This indicates that, the crystal composition of the bulk film is independent of the substrate type.

Figure 5(a) is the XRD spectra of thin films with varying composition deposited on sapphire substrate, Figure $5(\mathrm{~b})$ provides the spectra of films grown on silicon dioxide, and Figure 5(c) presents the spectra of films grown on silicon substrate. Peaks correlating with the (111), (220), (311) and (004) SiGeSn peaks were observed with varying intensities in all the XRD spectra irrespective of the composition, thickness, and the substrate. This implies that the deposited films are polycrystalline in nature and have a diamond cubic lattice structure [28]. It can be noted that the positions of the peaks shift with respect to the Ge peaks (as can be seen in Figures 5(d)-(f)). This is attributed to the fact that Si has a lower lattice constant $(5.43 \AA$ ) while Sn has a higher lattice constant $(6.46 \AA)$ with respect to $\mathrm{Ge}(5.66 \AA)$ causing an overall offset in the lattice constant of the ternary alloy based on the composition of Si and Sn. Runs 2 and 4 (low Sn concentrations) tend to offset the peaks to the right of pure Ge (111) while runs 1 and 3 (higher Sn concentrations) are seen to offset the peaks towards the left.

The polycrystallinity of the SiGeSn films on all the three substrates is explained by the synergetic effect of the chemical affinity of atomic $\mathrm{Si}$, Ge to atomic $\mathrm{O}$ and the Volmer-Weber growth (also known as the 3D island growth) reported by

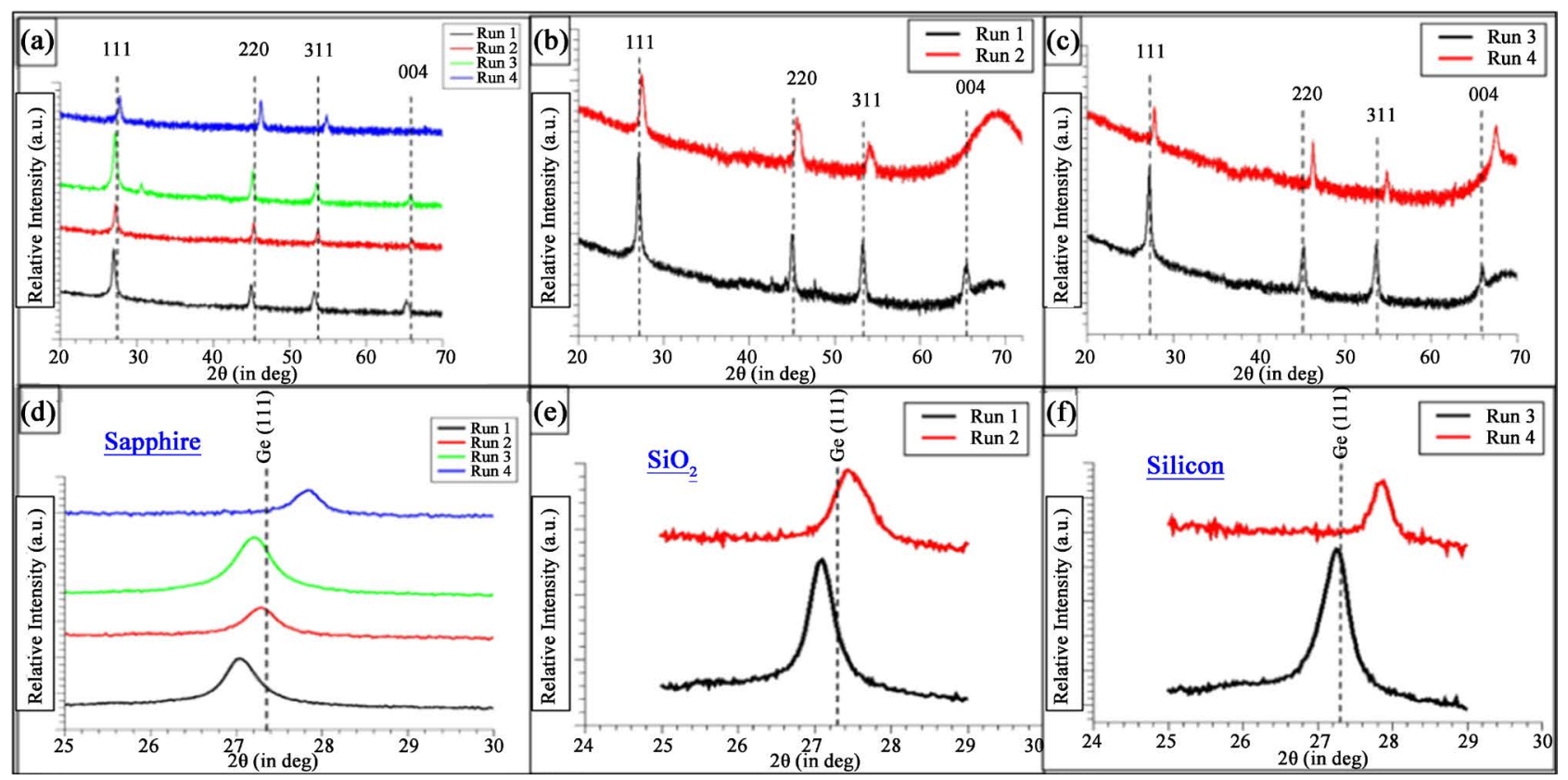

Figure 5. XRD spectra of: (a) SiGeSn films with varying composition on sapphire substrate; (b) SiGeSn films with varying composition on silicon dioxide substrate; (c) SiGeSn films with varying composition on silicon substrate; (d)-(f) Peak offsets with respect to $\mathrm{Ge}(111)$ for sapphire, $\mathrm{SiO}_{2}$, silicon substrates respectively. 
several researchers for multiple systems where the adatom $(\mathrm{Si}, \mathrm{Ge})$-adatom $(\mathrm{O})$ interactions are stronger than the adatom $(\mathrm{Si}, \mathrm{Ge}$ )-surface (substrate) interactions [32] [33]. The Volmer-Weber non-equilibrium growth is demonstrated in Figure 6 as explained by L. Miglio and A. Sassella [34]. This phenomenon occurs when:

$$
\gamma_{f}+\gamma_{i}>\gamma_{s}
$$

where $\gamma_{f}=$ surface free energy of the SiGeSn film $\left(\mathrm{J} / \mathrm{m}^{2}\right)$;

$\gamma_{i}=$ surface free energy of the substrate-SiGeSn film interface $\left(\mathrm{J} / \mathrm{m}^{2}\right)$;

$\gamma_{s}=$ surface free energy of the substrate $\left(\mathrm{J} / \mathrm{m}^{2}\right)$.

The vacuum established by the mechanical pump was $10^{-2}$ torr. Using the ideal gas equation shown below for the reactor volume of $0.023 \mathrm{~m}^{3}$, a chamber temperature estimate of $523 \mathrm{~K}$ and $\sim 21 \%$ of $\mathrm{O}_{2}$ abundance in the air and two atoms of oxygen per molecule, the number of oxygen atoms in the chamber at this pressure were estimated to be $\sim 10^{16}-10^{17}$.

$$
N=\frac{P * V}{k * T} * 0.21 * 2
$$

where $N$ is the number of oxygen atoms;

$P$ is the chamber vacuum attained before the introduction of the precursors $\left(\mathrm{kg} \cdot \mathrm{m}^{-1} \cdot \mathrm{s}^{-2}\right)$;

$V$ is the chamber volume $\left(\mathrm{m}^{3}\right)$;

$k=1.38 * 10^{-23} \mathrm{~m}^{2} \mathrm{~kg} \cdot \mathrm{s}^{-2} \cdot \mathrm{K}^{-1}$ is the Boltzmann constant;

$T$ is the chamber ambient temperature $(\mathrm{K})$.

Elemental $\mathrm{Si}$ and $\mathrm{Ge}$ atoms are formed as the disilane and germane precursors undergo cracking under plasma. During the initial growth phase of the film, these $\mathrm{Si}$ and $\mathrm{Ge}$ atoms readily react with the $\mathrm{O}$ atoms. The chemical reactions between $\mathrm{Si}$ and $\mathrm{Ge}$ atoms with atomic oxygen are outlined as below:

$$
\begin{aligned}
& \mathrm{Si}+2 \mathrm{O} \rightarrow \mathrm{SiO}_{2} \\
& \mathrm{Ge}+2 \mathrm{O} \rightarrow \mathrm{GeO}_{2}
\end{aligned}
$$

It is known that the contribution of $\mathrm{Sn}$ is minimal due to it being the solute and due to the lower reduction potential of its oxide $(-0.12 \mathrm{~V})$ compared to that

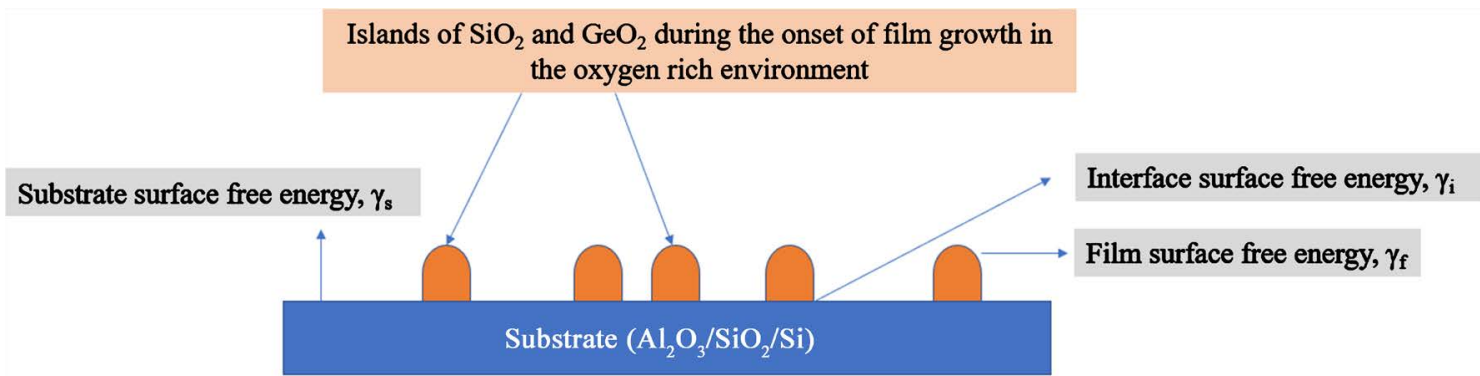

$$
\gamma_{\mathrm{f}}+\gamma_{\mathrm{i}}>\gamma_{\mathrm{s}}
$$

Figure 6. Volmer-Weber growth to explain the polycrystallinity of $\mathrm{SiGeSn}$ on sapphire, $\mathrm{SiO}_{2}$ and $\mathrm{Si}$ substrates in the oxygen abundant vacuum levels of PECVD chamber. 
of $\mathrm{Si}(-0.86 \mathrm{~V})$ and $\mathrm{Ge}(-0.18 \mathrm{~V})$.

These chemical reactions of $\mathrm{Si}$ and $\mathrm{Ge}$ atoms are more kinetically favorable compared to the adsorption of the $\mathrm{Si}$ or Ge atoms on to substrates due to the high affinity of these atoms to oxygen. The hence formed oxides of $\mathrm{Si}$ and Ge are then adsorbed on the substrate. These adsorbed amorphous oxides create islands on the substrate surface. The formation of these islands is expected to provide multiple nucleation sites for the $\mathrm{Si}, \mathrm{Ge}$, and $\mathrm{Sn}$ atoms during the deposition of the upcoming layers of film growth. Tensile and compressive stresses are created during the coalescence events of the islands and lattice stacking that result in high surface free energies of the SiGeSn film leading to the Volmer-Weber growth mechanism as explained in Figure 5 [34]. This phenomenon is theorized to be the reason for the onset of polycrystallinity.

It can be seen from Figure 5 that, for the films grown on sapphire, the (111) peak dominates the spectra in all films. This implies that most of the film has been deposited in the (111) orientation. This phenomenon has been previously reported for cubic structured films grown on c-plane sapphire substrates and is attributed to the low substrate free energy $\left(\gamma_{s}\right)$ provided by the (001) plane of sapphire that favors the formation of (111) SiGeSn crystal planes [34] [35] [36]. The (111) is also the strongest peak for the films deposited on silicon dioxide but it is not as dominant as for films deposited on (001) sapphire. This signals that the degree of randomness in crystal grain orientation for these films is higher than those deposited on sapphire. The grains being majorly (111) oriented has been previously recorded for cubic Ge and SiGe films grown on silicon dioxide [37] [38] [39] [40]. The films deposited on silicon substrate are seen to grow majorly in both the (111) and the (004) directions. The (004) orientation of the grains can be explained by the inclination of the film to grow epitaxially in (001) direction to minimize the interface free energy $\left(\gamma_{i}\right)$ [5] [6] [15]. Polycrystallinity occurs from the (111) orientation besides the (311) and the (220) orientations due to the formation of the $\mathrm{SiO}_{2}$ and the $\mathrm{GeO}_{2}$ islands on the $\mathrm{Si}$ surface as explained by chemical affinity combined with the Volmer-Weber growth mechanism.

To better visualize the preference of the crystal orientation of the grain domains for the different substrates, the areas under the curves for the (111), (220), (311) and (004) XRD peaks were calculated, added together and then the ratio of the area under each peak to the total area was obtained. Thus, the percentage of the domain growth in each orientation was obtained. This is shown in Figure 7.

The full width at half maximum (FWHM) of the (111) XRD peaks for the different films was obtained by fitting a modified Gaussian model and the values were then used to calculate the size of the grains using the Debye Scherrer formula [38] [41].

$$
t=\frac{k * \lambda}{\beta * \cos \theta}
$$

where $t$ is the average grain size, $k$ is the dimensionless constant shape factor, $\lambda$ is the X-ray wavelength (1.5405 $\AA$ for $\mathrm{Cu} \mathrm{K} \alpha$ line), $\beta$ is the FWHM (in radians) 


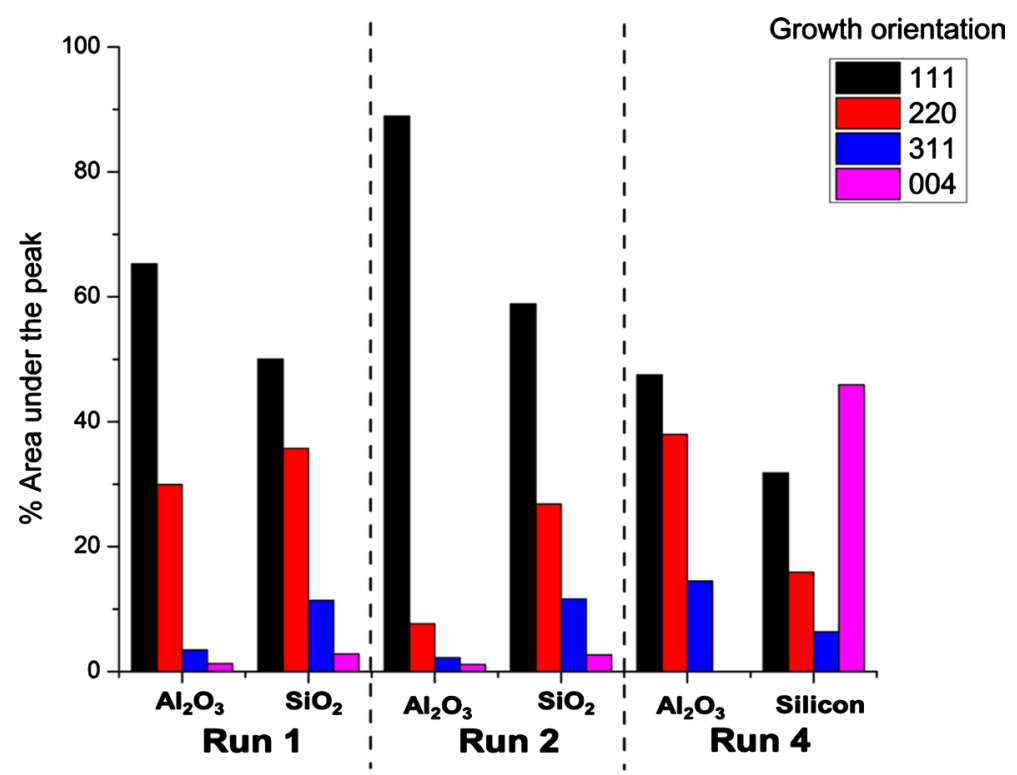

Figure 7. \% area under the XRD peaks for films deposited on different substrates with varying concentrations to depict the orientation preferences.

and $\theta$ is the Bragg angle. The value of $k$ was taken as 0.9 for simplification. It was observed that the grain sizes were in the region of $20-35 \mathrm{~nm}$ for the films. The small size of the domains implies that deposited films have a high density of grains. This is most likely brought about by the plasma assisted high cracking rate of the precursors. The grain size of the films grown on sapphire is larger than the films grown on silicon dioxide. This is explained by the large number of nucleation sites offered by the amorphous silicon dioxide. The broader distribution of lattice orientation on the $\mathrm{SiO}_{2}$ substrate makes it energetically more favorable to form smaller grains due to the added strain from multiple orientations. The grain size of the films grown on silicon is larger than the films grown on sapphire. The reason for this behavior is not yet known.

The lattice constant of the SiGeSn films was calculated using the position of (111) peak using Bragg's law with the assumption that the lattice is cubic in structure. The (111) peak was selected on account of its strong intensity in all the spectra. The relaxed lattice constant for a SiGeSn film with a particular composition was computed by using Vegard's law while considering the appropriate bowing parameter [10] [13] [41]. The lattice constant was calculated using the following equation:

$$
\begin{aligned}
a_{\mathrm{Si}_{y} \mathrm{Ge}_{1-x-y} \mathrm{Sn}_{x}}= & y * a_{S i}+(1-x-y) * a_{\mathrm{Ge}}+x * a_{\mathrm{Sn}}+y *(1-x-y) * b_{\mathrm{SiGe}} \\
& +x *(1-x-y) * b_{\mathrm{GeSn}}+x * y * b_{\mathrm{SiSn}}
\end{aligned}
$$

where $a_{\mathrm{Si}_{y} \mathrm{Ge}_{1-x-y} \mathrm{Sn}_{x}}$ is the lattice constant of the relaxed ternary alloy, $y, 1-x-y$ and $x$ are the concentration of $\mathrm{Si}, \mathrm{Ge}$ and $\mathrm{Sn}$ respectively in the ternary alloy, $a_{\mathrm{Si}}, a_{\mathrm{Ge}}$ and $a_{\mathrm{Sn}}$ are the lattice constants of $\mathrm{Si}, \mathrm{Ge}$ and $\mathrm{Sn}$ respectively with $a_{\mathrm{Si}}=5.431 \AA, a_{\mathrm{Ge}}=5.658 \AA$ and $a_{\mathrm{Sn}}=6.489 \AA, b_{\mathrm{SiGe}}=-0.26 \AA$, $b_{\mathrm{GeSn}}=-1.66 \AA$ and $b_{\mathrm{SiSn}}=0$. The obtained values of the lattice constants, 
from Bragg's law and Vegard's law, are given in Table 2.

Understanding the strain present in the films is of use as the band-gap of SiGeSn is dependent on the strain [19]. The difference in the lattice constant obtained by XRD and that calculated based on composition is used for strain analysis. The strain $\%$ is calculated by:

$$
\text { Strain } \%=\frac{a_{\text {Bragg }}-a_{\text {Vegard }}}{a_{\text {Vegard }}} * 100
$$

The obtained strain for the films deposited on the various substrates and with the corresponding Sn compositions is given in Figure 8. The strain is tensile in all the films irrespective of the chemical composition or substrate. Further, the strain is a strong function of the concentration of $\mathrm{Sn}$ in all the films. A higher $\mathrm{Sn}$

Table 2. Lattice constants obtained from Bragg's law and Vegard's law along with the chemical composition of the films.

\begin{tabular}{|c|c|c|c|c|c|c|c|}
\hline No. & $\mathrm{Si}(\mathrm{y})$ & $\mathrm{Ge}(1-\mathrm{x}-\mathrm{y})$ & $\operatorname{Sn}(x)$ & Substrate & $a_{\text {Bragg }}(\AA)$ & avegard $(\AA)$ & $\Delta \mathrm{a}(\AA)$ \\
\hline \multirow[t]{2}{*}{1} & 0.08 & 0.85 & 0.07 & Sapphire & 5.70 & 5.59 & 0.11 \\
\hline & & & & $\mathrm{SiO}_{2}$ & 5.69 & 5.59 & 0.10 \\
\hline \multirow[t]{2}{*}{2} & 0.26 & 0.71 & 0.03 & Sapphire & 5.62 & 5.54 & 0.09 \\
\hline & & & & $\mathrm{SiO}_{2}$ & 5.62 & 5.54 & 0.08 \\
\hline \multirow[t]{2}{*}{3} & 0.12 & 0.84 & 0.04 & Sapphire & 5.67 & 5.59 & 0.09 \\
\hline & & & & Silicon & 5.66 & 5.59 & 0.08 \\
\hline \multirow[t]{2}{*}{4} & 0.53 & 0.45 & 0.02 & Sapphire & 5.55 & 5.48 & 0.07 \\
\hline & & & & Silicon & 5.54 & 5.48 & 0.06 \\
\hline - & 0 & 1 & 0 & N/A (bulk) & 5.66 & 5.66 & 0 \\
\hline
\end{tabular}

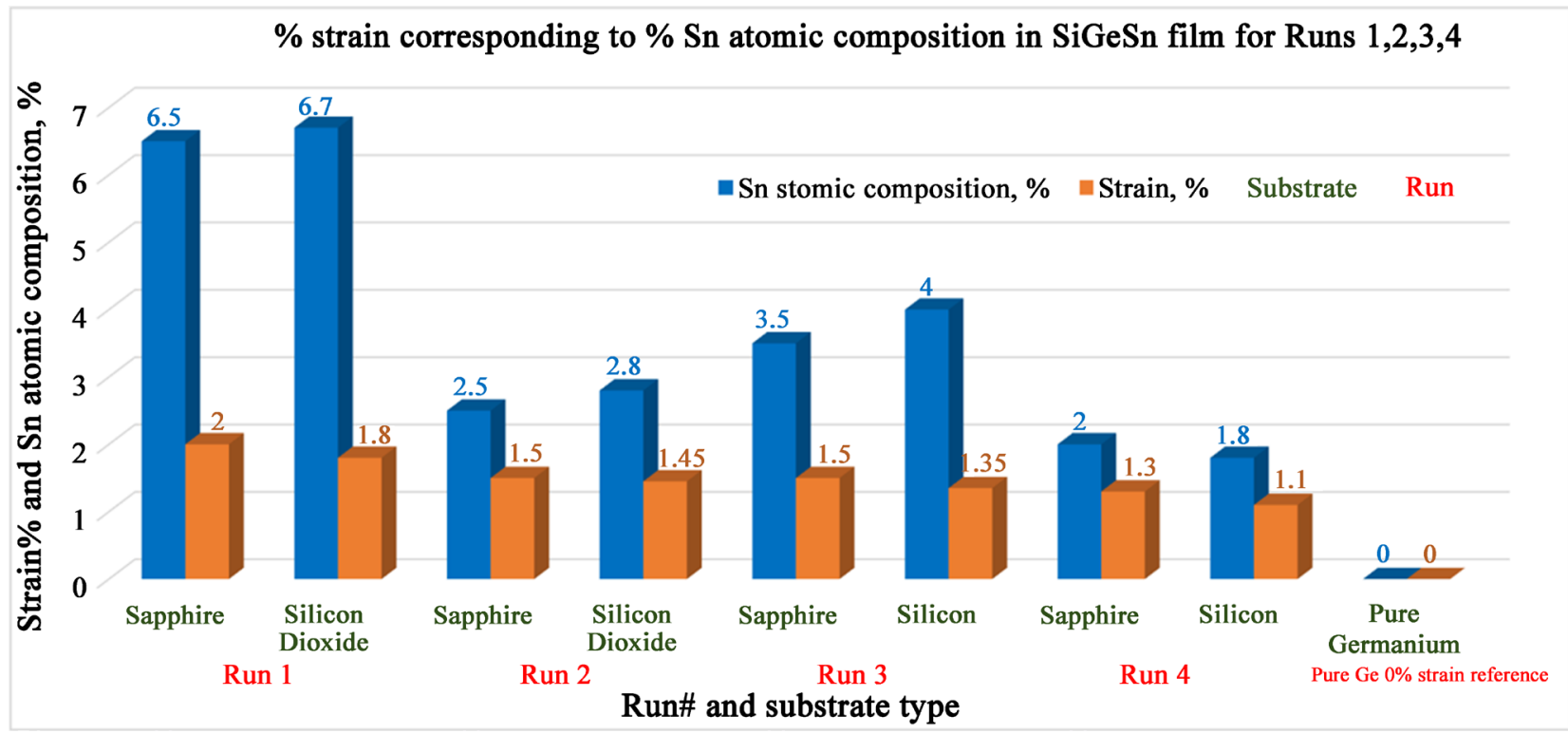

Figure 8. Lattice strain for SiGeSn films grown on various substrates by XRD and corresponding Sn atomic compositions in the films. 
content leads to a higher lattice strain while a lower Sn content induces less strain. This is attributed to the larger atomic radius of Sn compared to Ge [19] [25]. Also, for films with a given Sn composition, the strain is higher for films grown on sapphire as compared to films grown on silicon dioxide or silicon. The relatively lower strain when using silicon as a substrate is attributed to the smaller lattice mismatch between the ternary film and silicon compared to that of the ternary film and sapphire $\left(a_{\mathrm{Si}}=5.431 \AA\right.$ vs $a_{\text {Sapphire }}=4.75 \AA$ ). The relatively lower strain for films grown on silicon dioxide compared to sapphire is explained by the higher density of grains and smaller grain sizes in the films grown on silicon dioxide compared to those on sapphire. The higher density of grains on the $\mathrm{SiO}_{2}$ substrate results in the formation of larger grain boundaries and misfit dislocations which facilitate strain relief in the film grown on $\mathrm{SiO}_{2}$.

\section{Conclusions}

The effect of growth temperature and substrate type on the film properties of SiGeSn films deposited using a simplified plasma-enhanced non-UHV chamber were studied. Temperatures above $400^{\circ} \mathrm{C}$ resulted in $\beta$-Sn coalescence and phase separations. The lowest growth temperature of $350^{\circ} \mathrm{C}$ showed the highest $\mathrm{Sn}$ concentrations in the lattice with crystal homogeneity although a higher temperature of $380^{\circ} \mathrm{C}$ was seen to more than double the deposition rates due to higher cracking energies. The polycrystallinity of all the films was explained by a synergy effect between the chemical affinity of silicon and germanium atoms to oxygen and the 3D island mechanism of the SiGeSn film deposition. The type of the substrate was seen to have no impact on the bulk composition and the thickness of the SiGeSn films. However, it played a strong role in the grain size, lattice strain and the distribution of crystal orientation in the films. The amorphous substrate $\mathrm{SiO}_{2}$ was seen to broaden this distribution due to the presence of more nucleation sites for film growth. The (001) sapphire substrate resulted in a dominance of the (111) orientation whereas the silicon substrate led to a higher distribution of the (004) orientation.

Thus, the crystal orientation and grain sizes of the plasma-enhanced CVD-grown films can be modulated by the choice of the substrate. This learning could be applied to achieve desirable film properties for not just silicon photonics, but a wide variety of applications like thermo-electrics, transistors, waveguides, piezoelectrics, etc.

\section{Acknowledgements}

The authors acknowledge that the work reported here was funded by the US Department of Energy (Award \#DOE-STTR DE-SC0015164), NASA (STTR Contract\# 80NSSC18P2145.STTR 2018-I and National Science Foundation (grant nos. $1624842,1839485,1933742)$. We gratefully acknowledge the Eyring Materials Center for the Rutherford backscattering spectrometer and the high-resolution X-ray diffraction facilities at Arizona State University. We acknowledge Dr. Qing Hua Wang 
for the use of the Raman spectrometer.

\section{Conflicts of Interest}

The authors declare no conflicts of interest regarding the publication of this paper.

\section{References}

[1] Alharthi, B., Dou, W., Grant, P.C., Grant, J.M., Morgan, T., Mosleh, A., et al. (2019) Low Temperature Epitaxy of High-Quality Ge Buffer Using Plasma Enhancement via UHV-CVD System for Photonic Device Applications. Applied Surface Science, 481, 246-254. https://doi.org/10.1016/j.apsusc.2019.03.062

[2] Hashemi, P., Ando, T., Balakrishnan, K., Koswatta, S., Lee, K.-L., Ott, J.A., et al. (2017) MoHigh Performance PMOS with Strained High-Ge-Content SiGe Fins for Advanced Logic Applications. 2017 International Symposium on VLSI Technology, Systems and Application, Hsinchu, 24-27 April 2017, Article ID: 16947461. https://doi.org/10.1109/VLSI-TSA.2017.7942468

[3] Polzer, A., Gaberl, W., Swoboda, R., Zimmermann, H., Fedeli, J.M. and Vivien, L. (2010) A $10 \mathrm{~Gb} / \mathrm{s}$ Transimpendance Amplifier for Hybrid Integration of a Ge PIN Waveguide Photodiode. Silicon Photonics and Photonic Integrated Circuits II. 7719, Article ID: 77191N. https://doi.org/10.1117/12.854312

[4] Soref, R.A. and Perry, C.H. (1991) Predicted Band Gap of the New Semiconductor SiGeSn. Journal of Applied Physics, 69, 539-541. https://doi.org/10.1063/1.347704

[5] Sun, G., Soref, R.A. and Cheng, H.H. (2010) Design of an Electrically Pumped Si$\mathrm{GeSn} / \mathrm{GeSn} / \mathrm{SiGeSn}$ Double-Heterostructure Midinfrared Laser. Journal of Applied Physics, 108, Article ID: 033107. https://doi.org/10.1063/1.3467766

[6] Ranjan, R. and Das, M.K. (2016) Theoretical Estimation of Optical Gain in Tin-Incorporated Group IV Alloy-Based Transistor Laser. Optical and Quantum Electronics, 48, Article No. 201. https://doi.org/10.1007/s11082-016-0459-4

[7] Wang, H., Han, G., Liu, Y., Hu, S., Zhang, C., Zhang, J. and Hao, Y. (2016) Theoretical Investigation of Performance Enhancement in GeSn/SiGeSn Type-II Staggered Heterojunction Tunneling FET. IEEE Transactions on Electron Devices, 63, 303-310. https://doi.org/10.1109/TED.2015.2503385

[8] Shimura, Y., Iwamoto, K., Yokogawa, R., Tomita, M., Tatsuoka, H., Uchiyama, H. and Ogura, A. (2021) Thermal Conductivity and Inelastic X-Ray Scattering Measurements on SiGeSn Polycrystalline Alloy. Japanese Journal of Applied Physics, 60, Article No. SBBF11. https://doi.org/10.35848/1347-4065/abdb83

[9] Ozkan, C.S., Nix, W.D. and Gao, H. (1997) Strain Relaxation and Defect Formation in Heteroepitaxial $\mathrm{Si}_{1-\mathrm{x}} \mathrm{Ge}_{\mathrm{x}}$ Films via Surface Roughening Induced by Controlled Annealing Experiments. Applied Physics Letters, 70, 2247-2249.

https://doi.org/10.1063/1.118819

[10] Desjardins, P., Spila, T., Gurdal, O., Taylor, N. and Greene. J.E. (1999) Hybrid Surface Roughening Modes during Low-Temperature Heteroepitaxy: Growth of Fully Strained Metastable $\mathrm{Ge}_{1-\mathrm{x}} \mathrm{Si}_{\mathrm{x}}$ Alloys on $\mathrm{Ge}(001) 2 \times 1$. Physics Review B, 60, 15993-15998. https://doi.org/10.1103/PhysRevB.60.15993

[11] Fleurial, J.P. and Borshchevsky, A. (1990) Si-Ge-Metal Ternary Phase Diagram Calculations. Journal of the Electrochemical Society, 137, Article No. 2928.

https://doi.org/10.1149/1.2087101 
[12] Wirths, S., Buca, D. and Mantl, S. (2016) Si-Ge-Sn Alloys: From Growth to Applications. Progress in Crystal Growth and Characterization of Materials, 62, 1-39. https://doi.org/10.1016/j.pcrysgrow.2015.11.001

[13] Zheng, J., Wang, S., Zhou, T., Zuo, Y., Cheng, B. and Wang, Q. (2015) Single-Crystalline $\mathrm{Ge}_{1-\mathrm{x}-\mathrm{y}} \mathrm{Si}_{\mathrm{x}} \mathrm{Sn}_{\mathrm{y}}$ Alloys on $\mathrm{Si}$ (100) Grown by Magnetron Sputtering. Optical Materials Express, 5, 287-294. https://doi.org/10.1364/OME.5.000287

[14] Fisher, I., Wendav, T., Augel, L., Jitpakdeebodin, S., Oliveira, F., Benedetti, A. and Stefanov, S. (2015) Growth and Characterization of SiGeSn Quantum Well Photodiodes. Optics Express, 23, 25048-25057. https://doi.org/10.1364/OE.23.025048

[15] Asano, T., Terashima, T., Yamaha, T., Kurosawa, M., Takeuchi, W., Taoka, N., Nakatsuka, O. and Zaima, S. (2014) Epitaxial Growth and Crystalline Properties of $\mathrm{Ge}_{1-\mathrm{x}-\mathrm{y}^{-}}$ $\mathrm{Si}_{\mathrm{x}} \mathrm{Sn}_{\mathrm{y}}$ Ternary Alloys. 20147 th International Silicon-Germanium Technology and Device Meeting (ISTDM), 110, 159-160.

[16] Shimura, Y., Asano, T., Yamaha, T., Fukuda, M., Takeuchi, W., Nakatsuka, O. and Zaima, S. (2017) EXAFS Study of Local Structure Contributing to Sn Stability in $\mathrm{Si}_{\mathrm{y}} \mathrm{Ge}_{1-\mathrm{y}-\mathrm{z}} \mathrm{Sn}_{\mathrm{z}}$. Materials Science in Semiconductor Processing, 70, 133-138. https://doi.org/10.1016/j.mssp.2016.11.013

[17] Talochkin, A.B., Timofeev, V.A., Gutakovskii, A.K. and Mashanov, V.I. (2017) Sn-Inbduced Decomposition of SiGeSn Alloys Grown on Si by Molecular Beam Epitaxy. Journal of Crystal Growth, 478, 205-211. https://doi.org/10.1016/j.jcrysgro.2017.09.005

[18] Margetis, J., Ghetmiri, S.A., Du, W., Conley, B.R., Mosleh, A., Soref, R., Sun, G., Domulevicz, L., Naseem, H.A. and Yu, S.-Q. (2014) Growth and Characterization of Epitaxial $\mathrm{Ge}_{1-\mathrm{x}} \mathrm{Sn}_{\mathrm{x}}$ Alloys and Heterostructures Using a Commercial CVD System. ECS Transactions, 64, 711-720. https://doi.org/10.1149/06406.0711ecst

[19] Mosleh, A., Ghetmiri, S.A., Conley, B.R., Hawkridge, M., Benamara, M., Nazzal, A., Tolle, J., Yu, S.-Q. and Naseem, H.A. (2014) Material Characterization of $\mathrm{Ge}_{1-\mathrm{x}} \mathrm{Sn}_{\mathrm{x}}$ Alloys Grown by a Commercial CVD System for Optoelectronic Device Applications. Journal of Electronic Materials, 43, 938-946. https://doi.org/10.1007/s11664-014-3089-2

[20] Bauer, M., Ritter, C., Crozier, P.A., Ren, J., Menendez, J., Wolf, G. and Kouvetakis, J. (2003) Synthesis of Ternary SiGeSn Semiconductors on Si (100) via $\mathrm{Sn}_{\mathrm{x}} \mathrm{Ge}_{1-\mathrm{x}}$ Buffer Layers. Applied Physics Letters, 83, 2163-2165. https://doi.org/10.1063/1.1606104

[21] D’Costa, V.R, Tolle, J., Poweleit, C.D., Kouvetakis, J. and Menendez, J. (2007) Compositional Dependence of Raman Frequencies in Ternary $\mathrm{Ge}_{1-\mathrm{x}-\mathrm{y}} \mathrm{Si}_{\mathrm{x}} \mathrm{Sn}_{\mathrm{y}}$ Alloys. Physical Review B: Condensed Matter and Materials Physics, 76, Article ID: 035211.

[22] Gallaghar, J.D., Xu, C., Jiang, L., Kouvetakis, J. and Menendez, J. (2013) Fundamental Band Gap and Direct-Indirect Crossover in $\mathrm{Ge}_{1-\mathrm{x}-\mathrm{y}} \mathrm{Si}_{\mathrm{x}} \mathrm{Sn}_{\mathrm{y}}$. Applied Physics Letters, 103, Article ID: 202104. https://doi.org/10.1063/1.4829621

[23] Kouvetakis, J., Tolle, J., Matthews, J., Roucka, R. and Menendez, J. (2010) Si-Ge-Sn Technologies: From Molecules to Materials to Prototype Devices. ECS Transactions, 33, 615-628. https://doi.org/10.1149/1.3487592

[24] Mosleh, A., Alher, M., Cousar, L., Du, W., Ghetmiri, S.A., Pham, T., et al. (2015) Direct Growth of $\mathrm{Ge}_{1-\mathrm{x}} \mathrm{Sn}_{\mathrm{x}}$ Films on Si Using a Cold-Wall Utra-High Vacuum Chemical-Vapor-Deposition System. Frontiers in Materials, 2, Article No. 30. https://doi.org/10.3389/fmats.2015.00030

[25] Mosleh, A., Alher, M., Du, W. and Cousar, L. (2016) $\mathrm{Si}_{\mathrm{y}} \mathrm{Ge}_{1-\mathrm{x}-\mathrm{y}} \mathrm{Sn}_{\mathrm{x}}$ Films Grown on Si Using a Cold-Wall Ultrahigh-Vacuum Chemical Vapor Deposition System. Journal 
of Vacuum Science \& Technology B, 34, Article No. 011201. https://doi.org/10.1116/1.4936892

[26] Rouchka, R., Clark, A., Wilson, T., Thomas, T., Fuhrer, M., Ekins-Daukes, N., Johnson, A., Hoffman, R. and Begarney, D. (2016) Demonstrating Dilute-Tin Alloy SiGeSn for Use in Multijunction Photovoltaics. IEEE Journal of Photovoltaics, 6, 1025-1030. https://doi.org/10.1109/JPHOTOV.2016.2559785

[27] Vanjaria, J., Arjunan, A.C., Wu, Y., Tompa, G.S. and Yu, H. (2020) Epitaxial Ge Thin Film Growth on Si Using a Cost-Effective Process in Simplified CVD Reactor. ECS Journal of Solid State Science and Technology, 9, Article ID: 034008. https://doi.org/10.1149/2162-8777/ab80b0

[28] Banihashemian, S.F., Grant, J.M., Sabbar, A., Tran, H., Olorunsola, O., Ojo, S., Amoah, S., Mehboudi, M., Yu, S.-Q., Mosleh, A. and Naseem, H.A. (2020) Growth and Characterization of Low-Temperature $\mathrm{Si}_{1-\mathrm{x}} \mathrm{Sn}_{\mathrm{x}}$ on Si Using Plasma Enhanced Chemical Vapor Deposition. Optical Materials Express, 10, 2242-2253. https://doi.org/10.1364/OME.398958

[29] Vanjaria, J., Arjunan, A.C., Salagaj, T., Tompa, G.S., Yang, H., Houghton, T. and Yu, H. (2020) Growth of SiGeSn Thin Films Using Simplified PECVD Reactor towards NIR Sensor Devices. ECS Journal of Solid State Science and Technology, 9 , Article ID: 074001. https://doi.org/10.1149/2162-8777/abaeb2

[30] Vanjaria, J., Arjunan, A.C., Salagaj, T., Tompa, G.S. and Yu, H. (2020) PECVD Growth of Composition Graded SiGeSn Thin Films as Novel Approach to Limit Tin Segregation. ECS Journal of Solid State Science and Technology, 9, Article ID: 034009. https://doi.org/10.1149/2162-8777/ab80af

[31] Wang, L., Zhang, Y., Sun, H., You, J., Miao, Y., Dong, Z., Liu, T., Jiang, Z. and Hu, H. (2021) Nanoscale Growth of a Sn-Guided SiGeSn Alloy on Si (111) Substrates by Molecular Beam Epitaxy. Nanoscale Advance, 3, 997-1004.

https://doi.org/10.1039/D0NA00680G

[32] Friesen, C. and Thompson, C.V. (2002) Reversible Stress Relaxation during Precoalescence Interruptions of Volmer-Weber Thin Film Growth. Physical Review Letters, 89, Article ID: 126103. https://doi.org/10.1103/PhysRevLett.89.126103

[33] Lozovoy, K.A., Kokhanenko, A.P., Dirko, V.V., Akimenko, N.Yu. and Voitsekhovskii, A.V. (2019) Evolution of Epitaxial Quantum Dots Formed by Volmer-Weber Growth Mechanism. Crystal Growth \& Design, 19, 7015-7021.

https://doi.org/10.1021/acs.cgd.9b00820

[34] Miglio, L. and Sassella, A. (2005) Epitaxy. In: Bassani, F., Liedl, G.L. and Wyder, P., Eds., Encyclopedia of Condensed Matter Physics, Academic Press, Cambridge, 157-166. https://doi.org/10.1016/B0-12-369401-9/00692-6

[35] Park, Y., King, G.C. and Choi, S.H. (2008) Rhombohedral Epitaxy of Cubic SiGe on Trigonal C-Plane Sapphire. Journal of Crystal Growth, 310, 2724-2731. https://doi.org/10.1016/j.jcrysgro.2008.02.010

[36] Kim, H.J., Park, Y., Bae, H.B. and Choi, S.H. (2015) High-Electron-Mobility SiGe on Sapphire Substrate for Fast Chipsets. Advances in Condensed Matter Physics, 2015, Article ID: 785415. https://doi.org/10.1155/2015/785415

[37] Lager, G.A., Jorgensen, J.D. and Rotella, F.J. (1982) Crystal Structure and Thermal Expansion of $\alpha$-Quartz $\mathrm{SiO}_{2}$ at Low Temperatures. Journal of Applied Physics, 53, 6751-6756. https://doi.org/10.1063/1.330062

[38] Ohmura, T., Yamaha, T., Kurosawa, M., Takeuchi, W., Sakashita, M., Taoka, N., Nakatsuka, O. and Zaima, S. (2015) Mobility Behavior of Polycrystalline $\mathrm{Si}_{1-\mathrm{x}-\mathrm{y}} \mathrm{Ge}_{\mathrm{x}} \mathrm{Sn}_{\mathrm{y}}$ Grown on Insulators. Transactions of the Materials Research Society of Japan, 40, 
351-354. https://doi.org/10.14723/tmrsj.40.351

[39] Zhu, J.G., White, C.W., Budai, J.D., Withrow, S.P. and Chen, Y. (1995) Growth of Ge, $\mathrm{Si}$ and $\mathrm{SiGe}$ Nanocrystals in $\mathrm{SiO}_{2}$ Matrix. Journal of Applied Physics, 78, 4386-4389. https://doi.org/10.1063/1.359843

[40] Olivares, J., Rodriguez, A., Sangrador, J., Rodriguez, T., Ballesteros, C. and Kling, A. (1999) Solid-Phase Crystallization of Amorphous SiGe Films Deposited by LPCVD on $\mathrm{SiO}_{2}$ and Glass. Thin Solid Films, 337, 51-54. https://doi.org/10.1016/S0040-6090(98)01388-1

[41] Yamaha, T., Kurosawa, M., Ohmura, T., Takeuchi, W., Taoka, N., Nakatsuka, O. and Zaima, S. (2015) Effect of Sn on Crystallinity and Electronic Property of Low Temperature Grown Polycrystalline $\mathrm{Si}_{1-\mathrm{x}-\mathrm{y}} \mathrm{Ge}_{\mathrm{x}} \mathrm{Sn}_{\mathrm{y}}$ Layers on $\mathrm{SiO}_{2}$. Solid State Electronics, 110, 54-58. https://doi.org/10.1016/j.sse.2015.01.005 